\title{
Foudroyante nekrotisierende Fasziitis bei einem 47-jährigen Mann
}

Eike Gamon, Katharina Rahe, Henrik Beckschulze

\author{
Die nekrotisierende Fasziitis ist eine potenziell letale Entzündung des Weichteil- \\ gewebes, die sich durch rasant fortschreitende Gewebsnekrosen auszeichnet [1,2]. \\ Im Folgenden wird ein fulminanter Fall einer nekrotisierenden Fasziitis präsentiert. \\ Nach einem Bagatelltrauma nahm die Infektion einen hochgradigen septischen \\ Verlauf, der trotz maximaler Intensivtherapie und chirurgischer Intervention nicht \\ zu beherrschen war.
}

\section{Hintergrund}

Initial präsentieren sich Patienten mit nekrotisierender Fasziitis häufig mit unspezifischen Symptomen wie beispielsweise Fieber, typischerweise heftigen Schmerzen und Hautläsionen. Eine frühzeitige Diagnose und eine sich unmittelbar anschließende Therapie mit radikalem chirurgischem Vorgehen sowie intravenöser antibiotischer Therapie sind essenziell. Trotz optimierter interdisziplinärer Therapie liegt die Mortalität noch immer zwischen 15 und 46\% [3], unbehandelt sogar bei nahezu $100 \%$ [4].

Diverse Fälle von nekrotisierender Fasziitis wurden bereits in der internationalen Literatur beschrieben. Begünstigende Komorbiditäten im Zusammenhang mit nekrotisierender Fasziitis sind

- Diabetes mellitus,

- chronische Niereninsuffizienz,

- Adipositas,

- periphere arterielle Verschlusskrankheit,

- arterielle Hypertonie,

- intravenöser Drogenabusus sowie

- eine Immunsuppression [5].

\section{Der Fall}

\section{Vorgeschichte}

Der 47-jährige Patient stellte sich am Morgen des 1. Tages gegen 5:30 Uhr im Krankenhaus seines Wohnorts vor. Er hatte die vergangene Nacht aufgrund starker Schmerzen in der rechten Leiste sowie dem rechten Bein nicht schlafen können. Die Schmerzen hatten am Vorabend eingesetzt und rasch an Stärke zugenommen.
Anamnestisch waren keine Vorerkrankungen außer Heuschnupfen bekannt. Eine Dauermedikation bestand nicht, Voroperationen wurden ebenfalls verneint. Der Patient gab an, er habe am Vorabend gegen die Schmerzen einmalig Ibuprofen 400 mg eingenommen.

Am vorangegangenen (Feiertags-)Wochenende sei er mit Freunden auf Mallorca gewesen. An Unternehmungen hätte er nur partiell teilnehmen können, da er sich schon seit längerer Zeit etwas abgeschlagen fühle. Es wurde darüber hinaus über Phasen von Schüttelfrost berichtet. Auf einem Rückweg ins Hotel sei es während des Urlaubs zu einem Kollaps mit Sturz gekommen. Infektiöse Krankheitsbilder oder Fieber in den vorangegangenen Wochen wurden verneint, Verletzungen habe es nicht gegeben. Fremdanamnestisch wurde im Verlauf von der Ehefrau eine geringfügige Verletzung am rechten Fuß im Rahmen von Gartenarbeit angegeben. Diese lag aber etwa 4 Wochen zurück.

Nach seiner Rückkehr nach Deutschland am Vortag der Krankenhausaufnahme habe die Abgeschlagenheit weiter zugenommen. Es hätten zunehmende Schmerzen im rechten Bein und der rechten Leiste bestanden, die ihn vom Schlaf abgehalten hätten. Um seine Ehefrau nicht zu wecken, sei er in der Nacht in das Gästezimmer umgezogen. Bei weiterer Progredienz der Symptomatik habe er sich morgens zur Abklärung auf den Weg in die Klinik begeben.

\section{Beginn der Versorgung}

An jenem 1. Tag um 18:07 Uhr wird der zu unserer Klinik gehörende Rettungshubschrauber ( $\triangleright$ Abb. 1) in ein nahe gelegenes regionales Krankenhaus alarmiert. Er soll dort einen Patienten nach Reanimation übernehmen und in das zu unserer Klinik gehörende Herzkatheterlabor fliegen. 


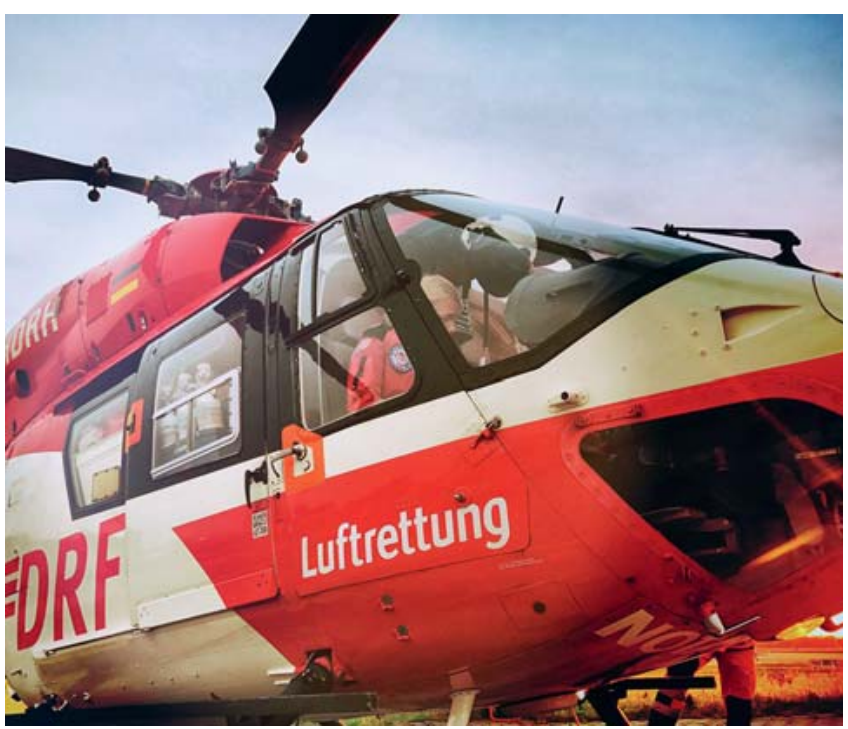

- Abb. 1 DRF Rettungshubschrauber Christoph Weser, Bremen.

Gegen 18:30 Uhr trifft das Rettungsteam auf der Intensivstation des abgebenden Krankenhauses ein und findet einen intubierten, beatmeten und hoch katecholaminpflichtigen 47-jährigen männlichen Patienten vor. Ein zentraler Venenkatheter ist gelegt worden, mehrfach sind arterielle Punktionsversuche unternommen worden, es kleben noch diverse sterile Tücher auf dem Patienten. Insgesamt wird der Eindruck des Patientenzimmers dominiert von der dort abgelaufenen Reanimation, sodass zu diesem Zeitpunkt die klinische Relevanz des umfangvermehrten rechten Oberschenkels untergeht. Ebenso lässt sich keine lokale Marmorierung am rechten Bein abgrenzen, da der Patient im Rahmen der hämodynamischen Zentralisation zwischenzeitlich an allen Extremitäten kutane Minderperfusionen aufweist.

In den Übergabegesprächen wird eine Auffälligkeit des rechten Oberschenkels erwähnt und diese als Grund der Vorstellung im Krankenhaus benannt. Ebenfalls wird über eine Reise mit einem längeren Flug berichtet, sodass differenzialdiagnostisch neben der rhythmogenen Genese für den Kreislaufstillstand eine Thrombose mit Lungenarterienembolie diskutiert wird. Allerdings lässt sich in der noch vor dem Transport durchgeführten transthorakalen Echokardiografie keine Rechtsherzbelastung erkennen. Ebenso sind die et $\mathrm{CO}_{2}$-Werte der Beatmung nicht auffällig. Valide Laborwerte gibt es bis auf einige unter Reanimation durchgeführte Blutgasanalysen nicht.

Es erfolgt eine Notfallversorgung und primär erfolgreiche Kreislaufstabilisierung des Patienten für den folgenden luftgebundenen Transport unter hochdosierter Katecholamin- und Volumentherapie. Dann startet der Hubschrauber mit dem Patienten um 19:00 Uhr und trifft um 19:25 Uhr im Herzkatheterlabor unserer Klinik ein.

\section{Aufnahmebefund}

Bei immer noch hochgradigem Verdacht auf eine kardiale Genese des Kammerflimmerns wird bei dem Patienten umgehend eine Herzkatheteruntersuchung und folgend auch eine weitere Echokardiografie durchgeführt. Sehr schnell ist klar, dass kein pathologischer Befund vorliegt, die linke wie rechte Koronararterie stellen sich unauffällig dar, es liegen keine Klappenvitien vor. Die linksventrikuläre Pumpfunktion entspricht dem erwarteten Befund unter hochdosierten Katecholaminen und wird vom Kardiologen als nicht pathologisch eingeschätzt.

Dem regelhaften diagnostischen Ablauf folgend wird der Patient unmittelbar einer CT-Diagnostik zugeführt und das Augenmerk auf den als auffällig beschriebenen rechten Oberschenkel gelenkt. Ebenfalls werden eine zerebrale Ursache für den Kreislaufstillstand (z. B. intrazerebrale/ Subarachnoidalblutung) und der schon früh geäußerte Verdacht der Lungenarterienembolie untersucht. Aufgrund der hoch instabilen Kreislaufsituation erfolgt unmittelbar nach der CT-Diagnostik die Verlegung auf die Intensivstation - noch bevor die CT-Aufnahmen durch den Radiologen abschließend beurteilt und bewertet worden sind.

\section{Verlauf}

Tag 1 - Fasziotomie des rechten Oberschenkels

Nach der Diagnostik wird der Patient gegen 21:30 Uhr auf die Intensivstation aufgenommen. Die Katecholamindosierungen liegen zu diesem Zeitpunkt bei Noradrenalin $0,9 \mu \mathrm{g} / \mathrm{kgKG} / \mathrm{min}$ und Adrenalin $0,8 \mu \mathrm{g} / \mathrm{kgKG} /$ min, dieses Therapieregime entspricht dem hausinternen Standard. Darunter sind die Blutdruckwerte mäßig stabil, der mittlere arterielle Druck liegt im Bereich von $60 \mathrm{mmHg}$.

Der radiologische Befund wird kurz darauf telefonisch mitgeteilt. Dort wird eine Imbibierung - also sinngemäß flüssigkeitsdurchtränktes Gewebe - beschrieben, vorwiegend in der Bauchdecke sowie dem rechten Oberschenkel mit vereinzelten Lufteinschlüssen. Der Verdacht einer Lungenarterienembolie bestätigt sich nicht, es zeigen sich keine Auffälligkeiten im CCT.

Bei hochgradigem Verdacht auf ein Kompartment-Syndrom des rechten Oberschenkels unter Beteiligung der Bauchdecke unklarer Genese erfolgt um 22:25 Uhr der erste operative Eingriff: eine Fasziotomie des rechten Oberschenkels, direkt auf der Intensivstation. Im operativen Bericht werden diffuse Einblutungen im Muskel, anhaltende seröse Sekretionen, aber eine grundsätzlich gut durchblutete vitale Muskulatur beschrieben. Umfangreiche mikrobiologische Abstriche werden gewonnen. Bei Aspiration während des Herz-Kreislauf-Stillstandes ist bereits im Herzkatheterlabor eine Antibiose mit Unacid $(3 \times 3 \mathrm{~g} / \mathrm{d})$ begonnen worden. Diese wird nun unter dem Verdacht auf ein hochgradig septiformes Bild auf Pipera- 
cillin/Tazobactam $(4 \times 4,5 \mathrm{~g} / \mathrm{d})$ und Clindamycin $(3 \times 600 \mathrm{mg} / \mathrm{d})$ eskaliert.

In den folgenden 5 Stunden muss bei steigenden Katecholamindosierungen neben der Volumensubstitution Empressin bis zur Dosis von $0,04 \mu \mathrm{g} / \mathrm{kgKG} / \mathrm{min}$ zur hämodynamischen Stabilisierung supplementiert werden. Parallel wird ein Stabilisierungsversuch mit 200 mg Hydrocortison unternommen.

Der völlig derangierte Säure-Basen-Status ist in der Übersicht der Blutgasanalysen zu erkennen ( $\triangleright$ Tab. 1). Unter kumulativ $600 \mathrm{ml}$ Natriumbikarbonat $8,4 \%$ und $40 \mathrm{ml}$ Tris-Puffer gelingt es, die Progredienz des Zustandes der metabolischen Azidose im Zusammenspiel mit einer Hyperventilation zu verlangsamen. So kann der Gesamtzustand stabilisiert werden.

Während dieses Zeitraums wird der Verdacht einer nekrotisierenden Fasziitis gestellt. Durch die erforderliche massive Volumensubstitution und die Verbrauchskoagulopathie des septischen Krankheitsbildes wird in dieser Phase der Einsatz von Blut- und Gerinnungsprodukten notwendig.

\section{Tag 2 - ausgedehnte Nekroseentfernung}

Um 3:50 Uhr wird nach erneuter Konsultation der chirurgischen Abteilung die Indikation zur großzügigen Sanierung der betroffenen Körperpartien gestellt und der Patient einer 10-stündigen Operation unterzogen. \ Abb. 2 und Abb. 3 zeigen das rechte Bein präoperativ vor ausgedehnter Nekroseentfernung (das Epigard des ersten Eingriffs ist noch zu sehen). > Abb. $\mathbf{4}$ zeigt den Patienten einige Stunden postoperativ mit aufgeklebten VAC-Verbänden.

Eine reine Amputation des betroffenen rechten Beines kommt zu diesem Zeitpunkt nicht infrage. Denn der Gewebsuntergang hat sich bereits auf das Becken, die Hüfte und die rechte Flanke ausgedehnt. Deshalb wird zu Beginn der OP der Entschluss gefasst, das betroffene Gewebe ausgedehnt zu sanieren und mit VAC-Verbänden zu versorgen. Allerdings werden im Laufe des operativen Eingriffs immer neue befallene Körperpartien entdeckt. Schon vorhandene VAC-Verbände müssen wieder entfernt und das Gewebe muss erneut debridiert werden.

Auf den postoperativen Aufnahmen ( $\bullet$ Abb. 4) ist einige Stunden nach der Operation gut zu erkennen: An den Rändern der Débridements bilden sich erneut Nekrosen aus (bläulich-livide Verfärbungen) als Zeichen des weiteren Fortschreitens des Gewebezerfalls.

Im OP-Bericht vermerkt das Chirurgenteam: „Im Bereich des gesamten rechten Beines, des Beckens und des distalen Rumpfes rechts zeigt sich ein unscharf begrenztes Erythem mit ausgedehntem Weichteilödem [...] extensi-

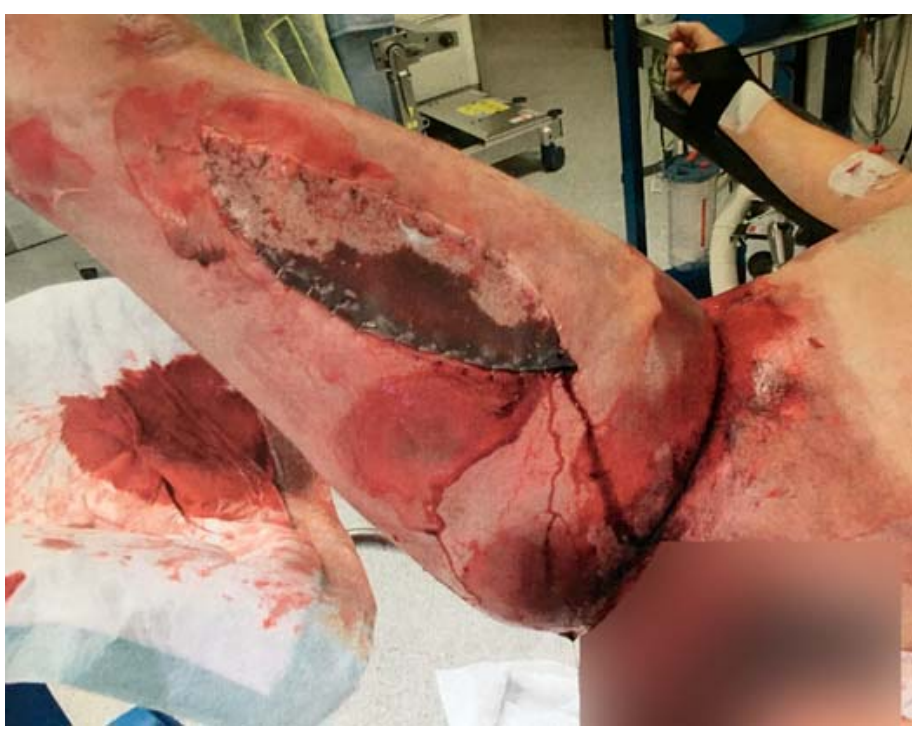

Abb. 2 Präoperativ mit Zustand nach Fasziotomie des rechten Oberschenkels.

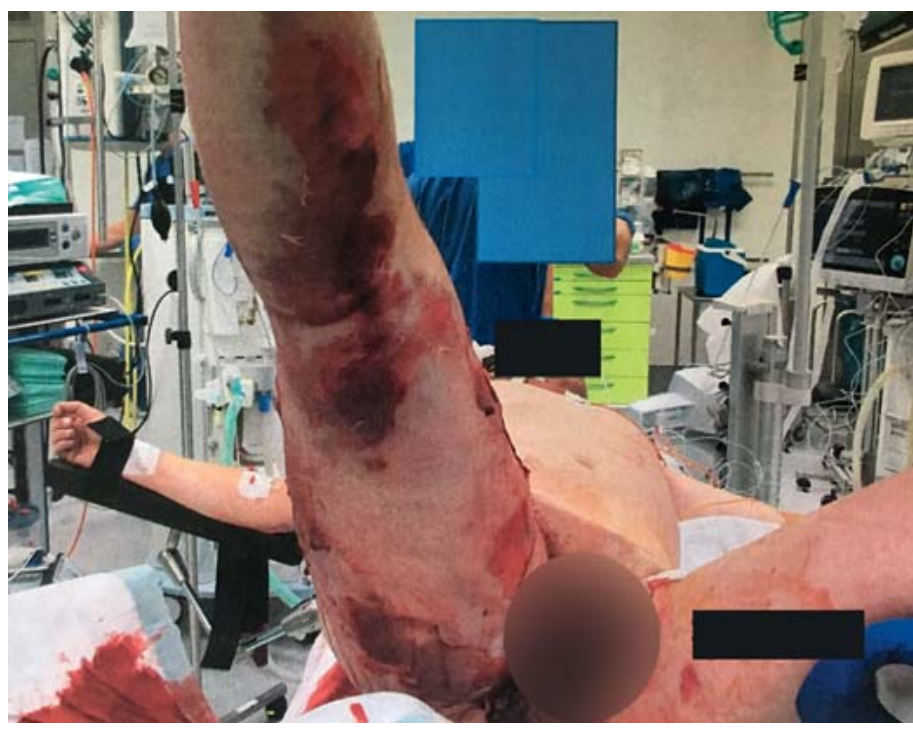

- Abb. 3 Rechtes Bein vor der 10-stündigen Nekroseentfernung.

ve Nekrosen der Faszie [...]. Die befallene Faszie wird radikal entfernt [...] Schnitterweiterung [...] bis in den Bereich der rechten Axilla [...]. Es zeigt sich, dass der Faszienbefall die gesamte rechte Rumpfwand bis in die Achselhöhle einnimmt. [...] Schnitterweiterung von der rechten Flanke nach distal [...] bis zum rechten Außenknöchel. [...] Die Ausbreitung der nekrotisierenden Fasziitis scheint im Verlauf der OP weiter foudroyant fortzuschreiten.“

Die Antibiose wird um 5 Mio. Einheiten Penicillin (einmalig im OP) und Linezolid $(3 \times 600 \mathrm{mg} / \mathrm{d})$ eskaliert. Gegen 13:30 Uhr am 2. Tag wird der Patient erneut auf die 


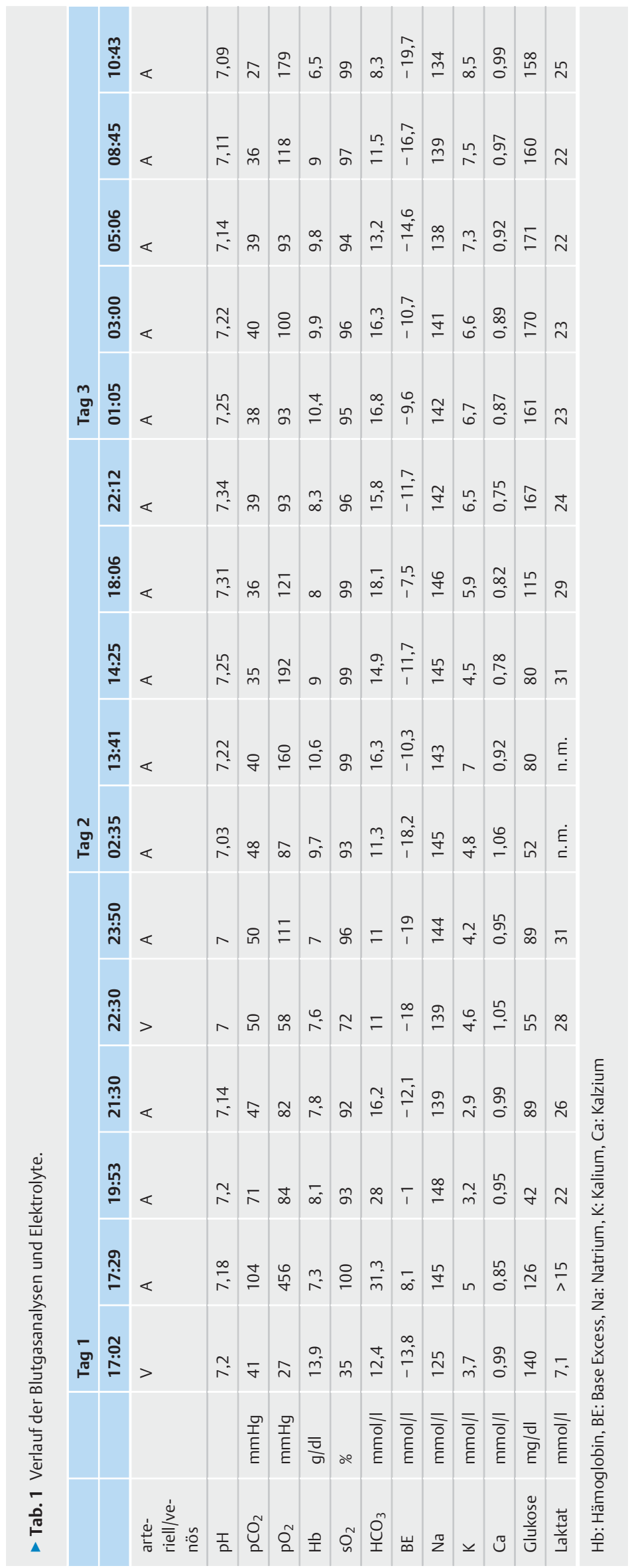



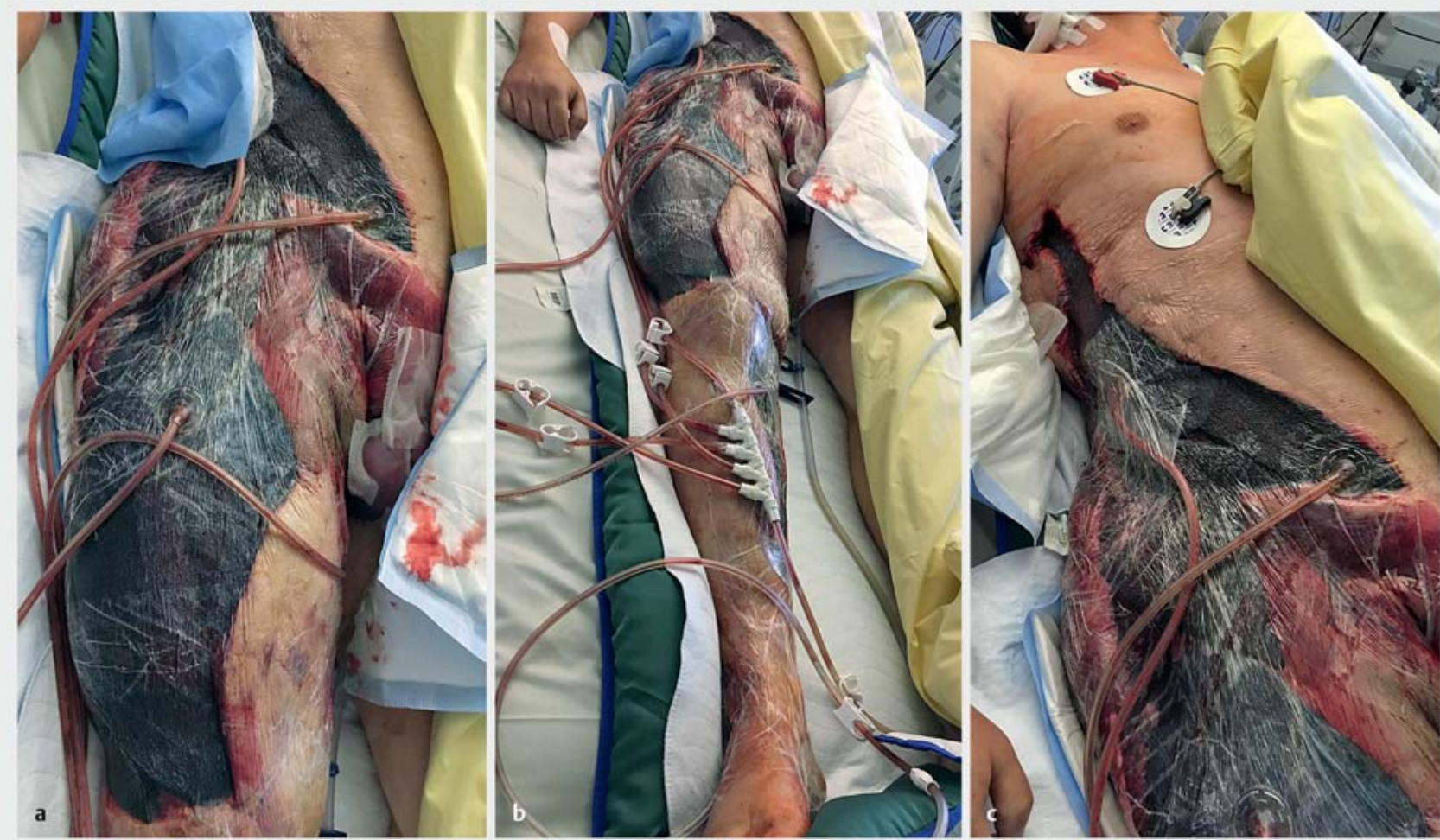

- Abb. 4 Postoperativer Situs mit VAC-Verbänden. a Rechter Oberschenkel, rechte Leiste. b Gesamtes rechtes Bein. c Rechte Flanke, Becken, Hüfte.

Intensivstation übernommen. Der Großteil der Muskulatur am rechten Bein und am rechten Rumpf ist entfernt worden. Über mehrere VAC-Pumpen wird das Wundsekret entfernt, der Patient verliert etwa 1500 ml Volumen in Form von Blut und Gewebsflüssigkeiten pro Stunde. Dies hat weitreichende Auswirkungen auf den Flüssigkeitshaushalt und die bereits vollständig entgleiste Gerinnung. Die Substitutionen von Volumen- und Gerinnungsprodukten werden fortgesetzt. Insgesamt werden während des 38-stündigen Aufenthalts auf unserer Intensivstation 42 Erythrozytenkonzentrate, 38 Frischplasmen, 4 Thrombozytenkonzentrate und 5000 Einheiten PPSB transfundiert.

Die Katecholamine liegen postoperativ bei Empressin $0,04 \mu \mathrm{g} / \mathrm{kgKG} / \mathrm{min}$, Noradrenalin $0,5 \mu \mathrm{g} / \mathrm{kgKG} / \mathrm{min}$ und Adrenalin $0,4 \mu \mathrm{g} / \mathrm{kgKG} / \mathrm{min}$. Hämodynamisch lässt sich der Zustand des Patienten damit nur mäßig beherrschen. Das hämodynamische Monitoring (Vigileo, Edwards Lifesciences $^{\mathrm{TM}}$ ) zeigt unter dieser medikamentösen Therapie völlig unzureichende Widerstände als Zeichen der septischen Vasoplegie und eine hyperdyname Hämodynamik im Rahmen des septischen Schocks ( $\bullet$ Abb. $\mathbf{5}$ und $\mathbf{6}$ ).

Wegen einer mittlerweile manifesten Hypothermie wird der Patient mit konvektiven Luftsystemen von oben und

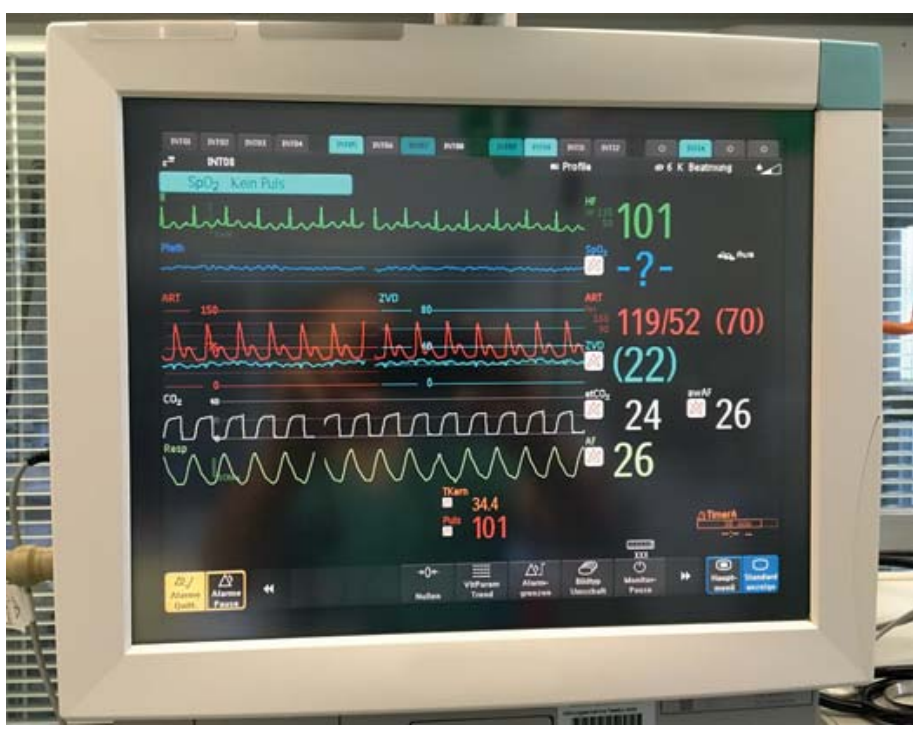

Abb. 5 Vitaldaten nach dem Débridement. 
- Tab. 2 Verlauf der Laborwerte.

\begin{tabular}{|c|c|c|c|c|c|c|c|c|c|c|}
\hline & & \multirow[b]{2}{*}{ Normbereich } & \multicolumn{2}{|l|}{ Tag 1} & \multicolumn{4}{|l|}{ Tag 2} & \multicolumn{2}{|l|}{ Tag 3} \\
\hline & & & 21:05 & $23: 58$ & $07: 23$ & $10: 45$ & $14: 00$ & $18: 20$ & $00: 29$ & 07:13 \\
\hline Quick & $\%$ & $70-130$ & $13-$ & $53-$ & $60-$ & $54-$ & $57-$ & $48-$ & $29-$ & $10-$ \\
\hline INR & & $2,0-4,0$ & $5,98+$ & $1,57-$ & $1,42-$ & $1,54-$ & $1,48^{-}$ & $1,69-$ & 2,72 & $8,19+$ \\
\hline pTT & s & $23-40$ & $>200+$ & $95+$ & $51+$ & $46+$ & $42+$ & $45+$ & $45+$ & $60+$ \\
\hline Fibrinogen & $\mathrm{g} / \mathrm{l}$ & $2,0-4,0$ & 2,44 & 2,26 & $1,96-$ & 2,16 & $1,93-$ & 2,48 & & \\
\hline Antithrombin & $\%$ & $80-130$ & $26-$ & 90 & $69-$ & & $55-$ & $68-$ & & \\
\hline D-Dimer & $\mu \mathrm{g} / \mathrm{l}$ & $<500$ & $9280+$ & & & & & 99 & & \\
\hline Leukozyten & $/ \mathrm{nl}$ & $4,0-10,0$ & 5,6 & 4,2 & $3,0-$ & $1,8^{-}$ & $2,8^{-}$ & & 4,0 & 6,4 \\
\hline Erythrozyten & /pl & $4,6-6,2$ & $2,29-$ & $2,09-$ & $3,15-$ & 2,84 & $3,16-$ & & $2,98^{-}$ & $3,46-$ \\
\hline Hämoglobin & $\mathrm{g} / \mathrm{dl}$ & $14,0-18,0$ & $7,3-$ & $6,6-$ & $9,0-$ & $8,3-$ & $9,6-$ & & $8,5^{-}$ & $10,0^{-}$ \\
\hline Hämatokrit & $\%$ & $42,0-52,0$ & $21,6-$ & $19,7-$ & $26,7-$ & $24,5^{-}$ & $27,8^{-}$ & & $24,7-$ & $29,0-$ \\
\hline Thrombozyten & $\mid \mathrm{nl}$ & $150-440$ & 157 & $100-$ & $95-$ & $31-$ & $75-$ & & $96-$ & 89- \\
\hline Kreatinin & $\mathrm{mg} / \mathrm{dl}$ & $0,67-1,17$ & $4,07+$ & $3,92+$ & $3,28+$ & $3,10+$ & $3,08+$ & & & $1,95+$ \\
\hline Harnstoff & $\mathrm{mg} / \mathrm{dl}$ & $19-44$ & $79,9+$ & $75,6+$ & $74,7+$ & & $70,8^{+}$ & & & $45,3+$ \\
\hline Bilirubin & $\mathrm{mg} / \mathrm{dl}$ & $0,1-1,2$ & & 0,77 & & & & & & \\
\hline GOT & $\mathrm{U} / \mathrm{I}$ & $<35$ & & $1708+$ & & & & & & $13842+$ \\
\hline GPT & $\mathrm{U} / \mathrm{I}$ & $<45$ & $437+$ & $805^{+}$ & $1878^{+}$ & & $1604+$ & & & $3984+$ \\
\hline GLDH & $\mathrm{U} / \mathrm{I}$ & $<6,4$ & & $230,4+$ & & & & & & \\
\hline$y-G T$ & $\mathrm{U} / \mathrm{I}$ & $<55$ & & 24 & & & & & & 48 \\
\hline CHE & $\mathrm{U} / \mathrm{I}$ & $4620-11500$ & & $3379-$ & & & & & & 5602 \\
\hline LDH & $\mathrm{U} / \mathrm{I}$ & $<248$ & & $1915+$ & & & & & & $8640+$ \\
\hline CK & $\mathrm{U} / \mathrm{I}$ & $<171$ & $11513+$ & $18239+$ & $35749+$ & $32555+$ & $30148+$ & & $51493+$ & $82290+$ \\
\hline Lipase & $\mathrm{U} / \mathrm{I}$ & $13-60$ & & 15 & & & & & & $297+$ \\
\hline CK-MB & $\mu \mathrm{g} / \mathrm{l}$ & $<4,87$ & $99,89+$ & $185,7+$ & $>300+$ & & $259,3+$ & & $360,2+$ & $259,3+$ \\
\hline CRP & $\mathrm{mg} / \mathrm{l}$ & $<5,0$ & $102,3+$ & $65,6+$ & $21,9+$ & & $22,0+$ & & & $53,6+$ \\
\hline PCT & $\mu \mathrm{g} / \mathrm{l}$ & & & $21,85+$ & & & & & & \\
\hline
\end{tabular}

INR: International normalized Ratio, pTT: partielle Thromboplastinzeit, GOT: Glutamat-Oxalacetat-Transaminase, GPT: Glutamat-Pyruvat-Transaminase, GLDH: Glutamatdehydrogenase, $y-G T$ : $y$-Glutamyltransferase, CHE: Cholinesterase, LDH: Laktatdehydrogenase, CK: Creatinkinase, CK-MB: Creatinkinase vom Myokardtyp, CRP: C-reaktives Protein, PCT: Procalcitonin

unten gewärmt. Eine CVVHDF (kontinuierliche venovenöse Hämodiafiltration) läuft mit maximaler Filtrationsleistung, um das durch die Rhabdomyolyse anfallende Kalium und die dadurch entstandene Hyperkaliämie zu eliminieren und die mittlerweile sistierende Nierenfunktion zu übernehmen ( $>$ Tab. 1 und 2 ).

In der interdisziplinären Visite, an der auch die Kollegen der Herzchirurgie teilnehmen, wird der Einsatz einer vaECMO (venoarterielle extrakorporale Membranoxygenierung) diskutiert, um dem sich anbahnenden Herz-Kreislauf-Versagen entgegenzuwirken. Schlussendlich finden sich bei dem Patienten zu diesem Zeitpunkt keine sicheren Punktionsorte für die Platzierung der erforderlichen Kanülen mehr. Die Option muss vor diesem Hintergrund verworfen werden. Ebenso wird kontrovers die Gesamtprognose auch unter einer möglichen ECLS-Therapie (extrakorporales Life Support System) diskutiert.
Tag 3 - weiteres Fortschreiten der Nekrosen

Um 5:00 Uhr fallen livide Verfärbung des rechten Oberarms und des Halses mit Emphysem und Lufteinschlüssen auf. Eine erneute CT-Diagnostik bestätigt den Verdacht des fortschreitenden Gewebezerfalls. Allerdings entfällt die Möglichkeit einer erneuten chirurgischen Sanierung seitens der Chirurgen bei nun vorliegender Beteiligung von Hals und Kopf. Während dieser CT-Untersuchung kommt es erstmalig im Verlauf zu Bradykardien und einer zeitweisen konsekutiven Asystolie. Nach kurzer Reanimationsdauer lässt sich ein erneuter Kreislauf etablieren.

Zurück auf der Intensivstation kann der steigende Kaliumspiegel weder durch den Einsatz der Nierenersatztherapie (CVVHDF) noch die parallele Gabe von Insulin und Kalzium unterbunden werden. Die Kaliumwerte übersteigen zu diesem Zeitpunkt 8 mmol/l ( $\triangleright$ Tab. 1). 


\section{ZUSAMMENFASSUNG}

\section{Chronologischer Verlauf}

Circa 3 Wochen vor Krankheitsbeginn:

- vermutetes Bagatelltrauma bei der Gartenarbeit

Circa 11 Tage vor Krankheitsbeginn:

- erstes Auftreten der Symptome mit Krankheitsgefühl und Schüttelfrost

Tag 1:

- 5:30 Uhr: Vorstellung in der Notaufnahme mit unspezifischem Krankheitsgefühl

- 17:00 Uhr: innerklinische Reanimation während der Diagnostik

- 18:30 Uhr: erster Kontakt des HubschrauberTeams mit dem reanimierten Patienten

- 19:25 Uhr: Übergabe des Patienten im Herzkatheterlabor

- 21:10 Uhr: CT-Diagnostik (CCT, CT Thorax, CT Abdomen, CT-Angiografie, CT HWS)

- 21:30 Uhr: Übernahme auf die Intensivstation

- 22:25 Uhr: Kompartment-Spaltung des rechten Oberschenkels auf der Intensivstation

- 23:20 Uhr: transösophageale Echokardiografie: biventrikuläre hochgradige Kardiomyopathie, keine Vitien, kein Perikarderguss

Tag 2:

- 3:50 Uhr: ausgedehnte 10-stündige Operation

- 13:30 Uhr: Wiederaufnahme auf der Intensivstation

- 14:00 Uhr: Beginn CVVHDF und intensivierte Insulintherapie

- 16:00 Uhr: interdisziplinäre Evaluation einer ECMO/ECLS

Tag 3:

- 5:00 Uhr: weitere livide Verfärbungen am rechten Oberarm und dem Hals

- 7:00 Uhr: erneute CT-Diagnostik, darunter Reanimationssituation

- 10:30 Uhr: zunehmende Herzrhythmusstörungen unter der progredienten Hyperkaliämie, folgend Reanimationspflicht, Einschwemmen eines transvenösen Pacers

- 11:06 Uhr: Der Patient verstirbt

Um 11:06 Uhr am 3. Tag - 37 Stunden und 36 Minuten nach Beginn unserer intensivmedizinischen Behandlung - verstirbt der Patient an einem Herzstillstand nach erneuten Bradykardien und folgender elektromechanischer Entkopplung durch die Hyperkaliämie. Auch das Einschwemmen eines transvenösen Schrittmachers wäh-

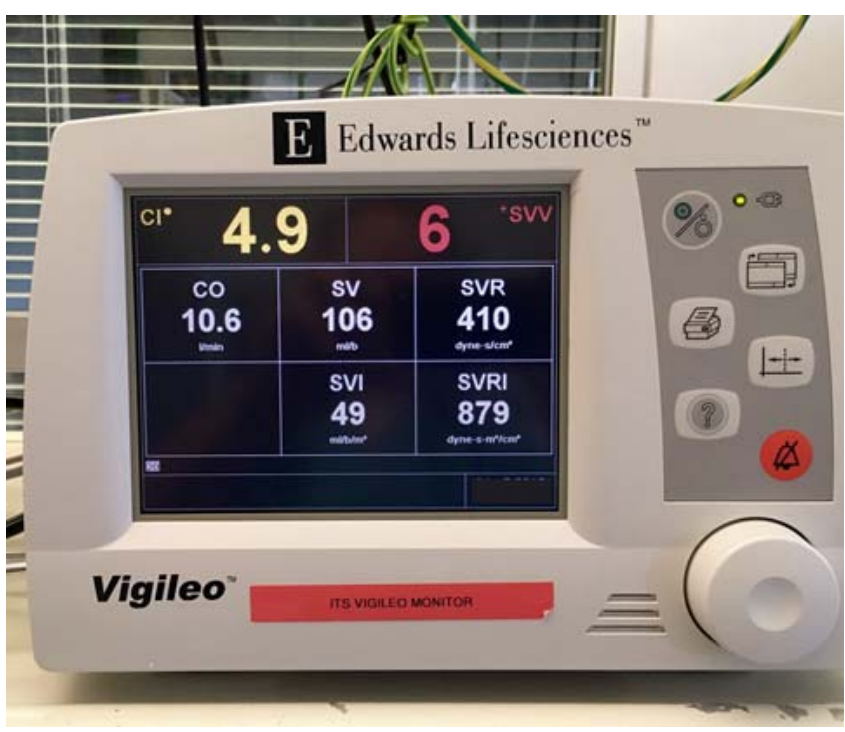

- Abb. 6 Hämodynamik (Vigileo) nach dem Débridement.

rend der Reanimation kann keine Herzaktionen mehr stimulieren. Die Reanimationsbemühungen bleiben auch nach über 45 Minuten frustran.

Im Nachhinein wird als verursachender Erreger Streptococcus pyogenes in sämtlichen Blutkulturen und Gewebsproben identifiziert.

\section{Diskussion}

Die nekrotisierende Fasziitis ist eine seltene Weichgewebsinfektion, die durch ein rasantes Fortschreiten von Weichteilnekrosen und unbehandelt von einer hohen Letalität gekennzeichnet ist [1,2].

Im Gegensatz zu diversen international publizierten Fallberichten wies der Patient im oben beschriebenen Fall keine für diese Erkrankung typischen Begleiterkrankungen auf. Dazu gehören z. B. Diabetes mellitus, chronische Niereninsuffizienz, Adipositas, periphere arterielle Verschlusskrankheit, arterielle Hypertonie, intravenöser Drogenabusus und eine Immunsuppression [5]. Auch hatte er keine regelmäßige Medikation und es bestand kein angeborenes Immundefizit. Auffällig ist daher der fulminante Verlauf der Erkrankung eines scheinbar gesunden und jungen Patienten; er ist in dieser Ausprägung in der Literatur selten zu finden. Um herauszufinden, was diese Fulminanz trotz offensichtlich fehlender Komorbiditäten bedingt hat, soll im Folgenden der Fall in seinen Einzelteilen diskutiert werden.

\section{Faktor „Zeit“}

Es gab eine Zeitverzögerung von 10 Tagen von Beginn der Symptomatik, über den stattgefundenen Kollaps im Hotel, bis zur Vorstellung im Krankenhaus. Diese scheint 
in diesem Zusammenhang mitverantwortlich für den fulminanten und schließlich letalen Verlauf zu sein. Noch weiter zurück lag die Entstehung der mutmaßlichen Eintrittspforte am Fuß bei der Gartenarbeit. So wurde von der Ehefrau ein Zeitraum von 3 Wochen zwischen der entstandenen Läsion und dem Symptombeginn beschrieben.

Der Patient wurde unserer Klinik aus einer Akutsituation heraus indirekt zugeführt, nämlich nach der Reanimation im regionalversorgenden Krankenhaus. Dadurch wurde die initiale Symptomatik der zu diesem Zeitpunkt fortgeschrittenen Infektion nicht unmittelbar erfasst. Eine Anamnese durch den Patienten oder seine Angehörigen war zu diesem Zeitpunkt nicht möglich. Allerdings bestand klinisch ein deutlich septiformes Bild, welches am ehesten den Kreislaufstillstand verursacht hatte. Ein SOFA- oder qSOFA-Score zur Identifikation einer Sepsis nach der aktuellen Leitlinie der Surviving Sepsis Campaign wurde von keinem der initial mit dem Patienten befassten Ärzte erhoben [6].

\section{Chirurgische Sanierung}

Die radikale Fasziotomie fand somit erst 9,5 Stunden nach der Aufnahme und 8 Stunden nach der ergebnislosen Herzkatheteruntersuchung statt. Bei den beschriebenen operativen Befunden und dem Ausmaß der Gewebsnekrosen war der Zeitverzug der operativen Versorgung sicherlich nicht ursächlich für den weiteren Verlauf. Er war allerdings auch nicht begünstigend für einen kurativen Ansatz. Eine frühere Diagnose war bei den unspezifischen Symptomen des zuvor gesunden Patienten jedoch kaum möglich gewesen. Ebenso bleibt spekulativ: Hätte eine frühere chirurgische Intervention den Verlauf maßgeblich verändert? Oder war zum Zeitpunkt des Reanimationsereignisses der Progress der Infektion bereits so weit fortgeschritten, dass der bis dato fulminante Verlauf nicht mehr reversibel war?

In vielen anderen beschriebenen Fällen einer nekrotisierenden Fasziitis wird eine meist initial betroffene Extremität frühzeitig amputiert und so versucht, ein Fortschreiten der Erkrankung zu verhindern. Die betroffenen Faszien imponieren nicht immer nekrotisch, sondern oft eher sulzig-imbibiert, sodass das volle Ausmaß des betroffenen Gewebes übersehen werden kann. In unserem Fall entschieden sich die chirurgischen Kollegen für ein maximal ausgedehntes Débridement, um so dem Patienten in diesem weit fortgeschrittenen Stadium eine Chance zu geben - auch unter Inkaufnahme einer langen OPZeit bei einem maximal instabilen Patienten.

Die Beschreibung dieses zeitlichen Verlaufs deckt sich mit der Erkenntnis mehrerer Studien: Die Zeitverzögerung bis zur chirurgischen Sanierung stellt den Hauptgrund für die Zunahme der Letalität bei der nekrotisierenden Fasziitis dar [7]. Dabei benannten Wong et al. schon 2003 ein 9-fach erhöhtes Risiko, an der nekrotisierenden Fasziitis zu versterben, wenn sich die Sanierung um mehr als 24 Stunden verzögerte [8].

\begin{abstract}
Merke
Der Faktor „Zeit“ scheint eines der wesentlichen Elemente einer erfolgreichen Behandlung der nekrotisierenden Fasziitis zu sein. Die schnelle Identifikation der Patienten ist essenziell für einen möglichen kurativen Therapieansatz.
\end{abstract}

\section{LRINEC Score}

Wong et al. entwickelten 2004 einen diagnostischen Score [9]. Dieser soll die Unterscheidung einer nekrotisierenden Fasziitis von anderen Weichgewebsinfektionen zu einem frühen Zeitpunkt erleichtern, sodass eine zielgerichtete Therapie früher eingeleitet werden kann (s. „Info - Diagnostischer Score“).

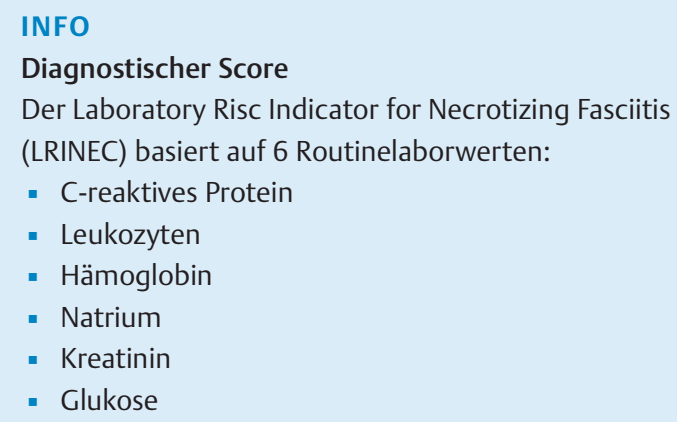

Er gibt die Wahrscheinlichkeit für das Vorliegen einer nekrotisierenden Fasziitis an und kann maximal den Wert 13 erreichen. Ab einem Wert von 6 sollte man an eine nekrotisierende Fasziitis denken, ab 8 sei die Wahrscheinlichkeit für eine nekrotisierende Fasziitis sehr hoch.

Der positive prädiktive Wert des Scores wurde mit 92\% angegeben. Allerdings wurden bereits Limitationen des Scores beschrieben, da er ausschließlich auf Laborparametern basiert. Die Komorbiditäten oder das Alter der Patienten und ihr klinischer Zustand werden nicht berücksichtigt $[10,11]$.

Im oben beschriebenen Fall konnte zum Aufnahmezeitpunkt des Patienten ein LRINEC-Wert von 4 ermittelt werden. Laut Definition ist dies kein erhöhtes Risiko für eine nekrotisierende Fasziitis (CRP 102,3 mg/l, Leukozyten 5,6/nl, Hämoglobin 7,3 g/dl, Natrium $139 \mathrm{mmol} / \mathrm{l}$, Kreatinin 4,7 mg/dl, Glukose $89 \mathrm{mg} / \mathrm{dl}$ ). Selbst die oben genannten limitierenden Faktoren hätten zu keinen weiterführenden Überlegungen neben dem Punktwert geführt: In Bezug auf Komorbiditäten und sein Alter gehörte der Patient keiner höhergradigen Risikogruppe an. 


\section{Intensivmedizinische Therapie}

Der dargestellte Fall unterstreicht neben der Fulminanz des Krankheitsverlaufes auch die intensivmedizinischen Herausforderungen in der Behandlung einer nekrotisierenden Fasziitis. Der Patient präsentierte sich nach Aufnahme auf die Intensivstation in einem schweren septischen Schockgeschehen. Neben der Katecholamintherapie und der invasiven Beatmung musste frühzeitig eine Nierenersatztherapie (CVVHDF) bei akutem Nierenversagen und exponentiell ansteigenden Kaliumwerten durch den fortschreitenden Zellzerfall eingeleitet werden. Trotz einer zusätzlich zur Nierenersatztherapie durchgeführten Gabe von Insulin und Kalzium konnte der Anstieg des Serumkaliums nicht beherrscht werden. Aufgrund der massiven Wundfläche und des konsekutiven Blut- und Flüssigkeitsverlustes war eine Massivtransfusion diverser Blut- und Gerinnungsprodukte erforderlich, da ebenfalls die Gerinnungssituation massiv kompromittiert war.

\section{Erreger und Antibiose}

Bezüglich des Erregerspektrums der nekrotisierenden Fasziitis liegt eine breite Fächerung vor in prinzipiell mono- oder polymikrobieller Form. Aktuell werden die Erreger in 4 Gruppen eingeteilt [4] (s. „Definition - Erregergruppen“).

\section{DEFINITION}

Erregergruppen

- Bei Typ I handelt es sich um polymikrobielle Infektionen mit obligat und fakultativ anaeroben Erregern, meist in Zusammenhang mit Vorerkrankungen wie beispielsweise Diabetes mellitus. Dieser Typ ist am häufigsten bei nekrotisierender Fasziitis nachzuweisen.

- Typ II stellen Infektionen durch beta-hämolysierende Streptokokken dar. Sie sind am häufigsten bei jungen und gesunden Patienten mit nekrotisierender Fasziitis nach kleinen Traumen an den Extremitäten zu finden. Zudem stehen sie im Zusammenhang mit NSAID (non-steroidal anti-inflammatory drugs).

- Typ III sind Infektionen mit Clostridien und gramnegativen Bakterien, oft nachzuweisen bei Drogenmissbrauch und bei Meeresfrüchteverzehr (insbesondere Aeromonas hydrophila).

- Typ IV beschreibt Pilzinfektionen, hauptsächlich durch Candida, bei immunsupprimierten Patienten.

Das Robert Koch-Institut (RKI) gibt auf seiner Homepage Informationen und Empfehlungen zur Therapie von Streptococcus-pyogenes-Infektionen, also für die Erregergruppe II, die auch im beschriebenen Fall vorlag [4, 12]. Einen Unterpunkt stellen schwere systemische
Streptococcus-pyogenes-Infektionen dar, u.a. Fasciitis necroticans. Bei dieser wird neben parenteralem Penicillin $G$ ebenfalls die Gabe von Clindamycin sowie von Immunglobulin-Präparaten empfohlen [12].

In der Hals-Nasen-Ohren-Heilkunde existiert eine S2-Leitlinie zur Antibiotikatherapie der Streptokokken-Infektionen an Kopf und Hals. Im Falle einer nekrotisierenden Fasziitis werden dort verschiedene Kombinationen von Breitspektrum-Antibiotika empfohlen [13].

Im oben beschriebenen Fall wurde breit kalkuliert antibiotisch therapiert: initial mit Piperacillin/Tazobactam und Clindamycin, intraoperativ mit Penicillin, später mit Linezolid, adäquat für beta-hämolysierende Streptokokken der Erregergruppe II. In allen Wundabstrichen sowie Kulturen aus Blut und Gewebe des Patienten wurden A-Streptokokken nachgewiesen.

\section{Hyperbare Sauerstofftherapie}

Diese Therapie wird schon seit über 20 Jahren diskutiert und bereits in einem Artikel aus dem Jahre 1998 im Deutschen Ärzteblatt dargestellt [14]. Aktuell im Jahr 2018 hat der Verband deutscher Druckkammerzentren e.V. (VDD) eine Übersicht über den aktuellen Stand der Literatur zur hyperbaren Sauerstofftherapie bei nekrotisierender Fasziitis herausgegeben [15]. Dabei soll die direkte Wirkung des Sauerstoffs auf die Bakterien diese abtöten und die körpereigene Abwehr auf Zellebene unterstützen. Voraussetzung hierfür ist die Therapie in einer Druckkammer mit deutlich erhöhten atmosphärischen Drücken. Im beschriebenen Fall hätte die Organisation eines freien Druckkammerplatzes für den Patienten und eine Verlegung in ein Zentrum viel Zeit gekostet. Laut der Liste der Gesellschaft für Tauch- und Überdruckmedizin (Stand 01.01.2018) wären in Deutschland nur 3 Standorte mit Druckkammern und Intensivbetten infrage gekommen: Stuttgart, Ulm und Regensburg [16]. Darüber hinaus hätte der Patient den Transport in dem zu diesem Zeitpunkt vorherrschenden instabilen Zustand höchstwahrscheinlich nicht überlebt.

\footnotetext{
Merke

Neben der Infektionsbekämpfung durch die kalkulierte Gabe von Antibiotika und die chirurgische Sanierung der betroffenen Gewebsteile ist die hyperbare Sauerstofftherapie eine weitere Therapieoption.

\section{Immunglobulin G}

Interessant erscheinen Forschungsergebnisse einer wissenschaftlichen Arbeitsgruppe des Helmholtz-Zentrums für Infektionsforschung (HZI) in Zusammenarbeit mit Universitätsmedizinern aus Schweden, Norwegen und Dänemark: Sie untersuchten die Wirkung von spezifischem Immunglobulin $\mathrm{G}$ in der frühen Infektionsphase von Streptococcus pyogenes im Zusammenspiel mit dem körpereigenen Immunsystem des Patienten [17].
} 


\section{Merke}

Die Studienergebnisse deuten darauf hin, dass die Gabe von Immunglobulin G durchaus den Erkrankungsverlauf entscheidend positiv beeinflussen kann. Hat jedoch die Gewebsnekrose schon begonnen, ist der Zellzerfall nicht mehr aufzuhalten [17].

Diese Therapie ist noch nicht flächendeckend verfügbar, sodass Kliniken ohne universitäre Strukturen keine Möglichkeit haben, kurzfristig solch eine Therapie bei ihren Patienten anzuwenden. Angemerkt sei hier, dass die Therapie mit Immunglobulinen - ähnlich wie die hyperbare Sauerstofftherapie - nicht neu ist. Auch sie wurde bereits vor über 20 Jahren als mögliche Therapieoption bei nekrotisierender Fasziitis diskutiert, aber es gibt noch keine flächendeckende etablierte Therapieempfehlung [14].

\section{Fazit}

Diese Kasuistik beschreibt den fulminanten Verlauf einer nekrotisierenden Fasziitis bei einem 47-jährigen Patienten, ausgelöst durch Streptococcus pyogenes, mit mutmaßlicher Infektion über eine Bagatellverletzung. Sie zeigt eindrucksvoll, welchen Weg die Erkrankung nehmen kann, wenn ein bestimmter Zenit überschritten und zu spät mit der chirurgischen und antibiotischen Therapie begonnen wird - anscheinend unabhängig von Komorbidität und Alter. Gleichfalls hilflos erscheint dann die Intensivmedizin.

\section{KERNAUSSAGEN}

- Ein kurativer Ansatz ist bei der nekrotisierenden Fasziitis nur in der Frühphase der Erkrankung durch eine radikale chirurgische Sanierung möglich. Bei bereits fortgeschrittenem Gewebezerfall ist die Prognose ungünstig.

- Mögliche Therapieoptionen neben einer begleitenden antibiotischen Therapie (hyperbare Sauerstofftherapie und Immunglobulintherapie) sind nicht für jede Klinik verfügbar. Bei deutlich fortgeschrittenem Krankheitsverlauf sind auch sie nicht mehr effektiv und realisierbar.

- Wie bei anderen fulminanten Krankheiten sollte versucht werden, suffizientere Scoring-Möglichkeiten zu finden, um betroffene Patienten frühzeitiger zu identifizieren, z. B. anhand der aktuellen Sepsiskriterien bzw. qSOFA.

- Wünschenswert wäre zudem eine spezifische interdisziplinäre Leitlinie für die Therapie der nekrotisierenden Fasziitis in Deutschland.

- Darüber hinaus sollten Ärzte und nichtärztliches Personal für dieses seltene Krankheitsbild sensibilisiert werden, um betroffene Patienten schnell erkennen und rechtzeitig eine gerichtete Therapie einleiten zu können.
Interessenkonflikt

Die Autoren geben an, dass kein Interessenkonflikt besteht.

Autorinnen/Autoren

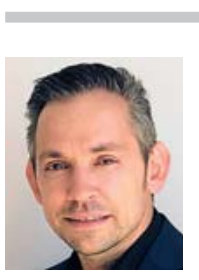

\section{Eike Gamon}

Dr. med., Jahrgang 1977. Studium der Humanmedizin 2005-2011 am UKE Hamburg. 2017 Facharzt für Anästhesiologie, 2018 Facharzt für Anästhesiologie, Zusatzbezeichnung anästhesiologische Intensivmedizin. Seit 2018 Oberarzt der Klinik für Anästhesiologie, Intensivmedizin und Notfallmedizin im Klinikum Links der Weser Bremen. Schwerpunkte: Intensivmedizin, Notfallmedizin.

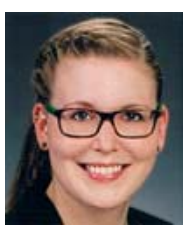

\section{Katharina Rahe}

Dr. med., Jahrgang 1988. Studium der Humanmedizin 2008-2014 an der MH Hannover. Seit 2015 Facharztausbildung Anästhesiologie, Assistenzärztin an der Klinik für Anästhesiologie, Marienhospital Osnabrück.

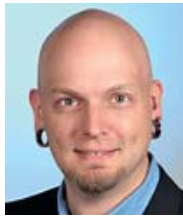

\section{Henrik Beckschulze}

Jahrgang 1989. Studium der Humanmedizin 2008-2015 an der Uni Würzburg. Seit 2016 Facharztausbildung Anästhesiologie, Assistenzarzt an der Klinik für Anästhesiologie, Intensivmedizin und Notfallmedizin im Klinikum Links der Weser Bremen.

\section{Korrespondenzadresse}

\section{Dr. med. Eike Gamon}

Klinikum Links der Weser

Anästhesiologie, Intensivmedizin, Notfallmedizin

Senator-Wessling-Straße 1

28277 Bremen

e.gamon@mail.de

\section{Literatur}

[1] Kueckelhaus M, Hirsch T, Lehnhardt M et al. Necrotizing fasciitis of the upper and lower extremities. Chirurg 2017; 88: 353366

[2] Castleberg E, Jenson N, Dinh VA. Diagnosis of necrotizing fasciitis with bedside ultrasound: the STAFF exam. West J Emerg Med 2014; 15: 111-113

[3] Puvanendran R, Huey JCM, Pasupathy S. Necrotising fasciitis. Can Fam Physician 2009; 55: 981-987

[4] Misiakos EP, Bagias G, Patapis P et al. Current concepts in the management of necrotizing fasciitis. Front Surg 2014; 1: 36. doi:10.3389/fsurg.2014.00036

[5] Goh T, Goh LG, Ang CH et al. Early diagnosis of necrotizing fasciitis. Br J Surg 2014; 101: e119-e125

[6] Rhodes A, Evans LE, Alhazzani W et al. Surviving Sepsis Campaign: International Guidelines for Management of Sepsis and Septic Shock: 2016. Intensive Care Med 2017; 43: 304-377

[7] Sarani B, Strong M, Pascual J et al. Necrotizing fasciitis: Current concept and review of the literature. J Am Coll Surg 2009; 208: 279-288 
[8] Wong $\mathrm{CH}$, Chang HC, Pasupathy S et al. Necrotizing fasciitis: clinical presentation, microbiology, and determinants of mortality. J Bone Joint Surg Am 2003; 85: 1454-1460

[9] Wong CH, Khin LW, Heng KS et al. The LRINEC (Laboratory Risk Indicator for Necrotizing Fasciitis) score: a tool for distinguishing necrotizing fasciitis from other soft tissue infections. Crit Care Med 2004; 32: 1535-1541

[10] Bechar J, Sepehripour S, Hardwicke J et al. Laboratory risk indicator for necrotising fasciitis (LRINEC) score for the assessment of early necrotising fasciitis: a systematic review of the literature. Ann R Coll Surg Engl 2017; 99: 341-346

[11] Cai Y, Gan Y, Yu C et al. A successful treatment of necrotizing fasciitis following the surgery of distal radius plate removal. A case report and literature review. Medicine 2018; 97: e0305

[12] Robert Koch-Institut. RKI-Ratgeber Infektionskrankheiten Merkblätter für Ärzte: Infektionen durch Streptococcus pyogenes. 2008. Im Internet: http://www.rki.de/DE/Content/Infekt/EpidBull/Archiv/2008/42/Art_01.html; Stand: 11.03.2019

[13] Deutsche Gesellschaft für Hals-Nasen-Ohren-Heilkunde, Kopfund Hals-Chirurgie. AWMF Leitlinie Nr. 017/066. Antibiotikatherapie der Infektionen an Kopf und Hals. 2010. Im Internet: http://www.awmf.org/uploads/tx_szleitlinien/017-066_S2_ Antibiotikatherapie_der_Infektionen_an_Kopf_und_Hals_ abgelaufen.pdf; Stand: 11.03.2019
[14] Podbielski A, Rozdzinski E, Wiedeck H et al. Gruppe-A-Streptokokken und die nekrotisierende Fasziitis. Dtsch Arztebl 1998; 95: A-414/B-350/C-326

[15] Verband Deutscher Druckkammerzentren VDD e.V. Die Hyperbare Sauerstofftherapie Adjuvant zur Behandlung von Nekrotisierender Fasciitis Fournier Gangrän. 2018. Im Internet: http://www.vdd-hbo.de/wp-content/uploads/2014/10/Nekrotisierende-Fasciitis_Fournier_HBO-Info-Ärzte_VDD.pdf; Stand: 11.03 .2019

[16] Gesellschaft für Tauch- und Überdruckmedizin e.V. Therapieeinrichtungen. Januar 2019. Im Internet: http://www.gtuem. org/1210/Druckkammer/24h-Dienst-Intensiv; $\quad$ Stand: 11.03.2019

[17] Babbar A, Bruun T, Hyldegaard O et al. Pivotal role of preexisting pathogen-specific antibodies in the development of necrotizing soft-tissue infections. J Infect Dis 2018; 218: 44-52. doi:10.1093/infdis/jiy110

Bibliografie

DOI https://doi.org/10.1055/a-0808-2548

Anästhesiol Intensivmed Notfallmed Schmerzther 2019; 54: 365-375 @ Georg Thieme Verlag KG Stuttgart · New York ISSN 0939-2661 